\title{
DE LA EVOLUCION DE LAS ESPECIES A LA EVOLUCION DE LAS CIENCIAS
}

\author{
MANUEL G. DONCEL
}

\section{SUMMARY}

In the ocassion of Darwin's centenary, toulmin's evolutive epistemology in stressed, within other modern epistemological currents, which are considered as fixist or evolutive in character. This epistemology distinguishes different levels of scienctifical change, and uses the Darwinian model to explain continuity and change in the scientifical disciplines. The dynamical concept of rationality which emerges from this study, can be useful for teaching sciences in a more humanistic way.

\section{DE LA EVOLUCION DE LAS ESPECIES A LA EVOLLICION DE LAS CIENCIAS}

Acabamos de clausurar el aho jubilar de Charles Dar. win. Sus multiples celebraciones nos han recordado una y otra vez el modelo explicalivo con que Darwin pretendia dar razón de la evolución de las especies, a base de variaciones al azar y selección natural. Con cien ahos de perspectiva este modelo explicativo aparece triunfance y limitado. Ha triunfado al justificar la conjetura - gratuita en tiempos de Darwin- de la heredabilidad de las variaciones, que hoy entendemos neodarwinianamente como mulaciones al azar del código genético. Aparece limitado en su excesiva simplicidad y hoy reviste modalidades mucho más comple. jas, cuando intenta explicar las transformaciones biológicas más radicales. Pero es un hecho que ese modeto explicativo ha trascendido las fronteras de la evolución biologica, para aplicarse a la evolución cosmica y aun a la evolución de las ciencias. Sin pretender absolutizarlo, ni considerarlo como explicación última de la realidad, vamos a ver cómo este modelo explicativo darwiniano puede ifuminar la moderna epistemologia evolutiva.

\section{EPISTEMOLOGIAS FIXISTAS Y EVOLUTIVAS}

La epistemologia, o reflexión sobre ef valor y los timites del conocimiento cientifico, ha sufrido muchos vaivenes en este último siglo. El antiguo inductivismo de Francis Bacon ${ }^{1}$ creia edificar las teorias cientificas sobre la base solida de los hechos rigurosamente establecidos. Asi habia pretendido demostrar Newton la gravitación universal y Ampère su "Teoria electrodi. namica deducida exclusivamente de la experiencia"' Tal epistemologia inductivista daba una visión fixista de las ciencias. Estas consisten para ella en construcciones rigurosas, indestructibles. El progreso histórico de las ciencias solo puede realizarse por acumulación, levantando más y más edificios, unos junco a otros o sobre otros, sin modificar para nada la estruc. lura interna de lus anteriores. Pero en la primera de. cena de nuestro siglo, Fisicos como Pierre Duhem ${ }^{3}$ y Henri Poincare a ambos muy sensibles a las transformaciones historicas de la fisica, inı rodujeron el llamado convencionalismo. Según el, las teorias no pueden probarse por inducción. Más bien se aceptan por convención, por ser sencillas y explicar mejor un conjunto de hechos. A lo largo de la historia tales convenciones pueden cambiar, y ser razonablemente sustitui. dads por orras aún más generales o más sencillas. Tal epistemologia convencionalista estaba pues abierta a una vision evoltuliva de las ciencias.

Pero desde los años 20 de nuestro siglo se fue impo. niendo otra vex una visión fixista de las ciencias. Es la visión del posirivismo logico del circulo de Vienas y de la Escuela de Beriin. Obsesionados por el terror a la especulación metafisica y escandilados por los triun. fos de la formalización lógica de las matemáticas, proyectan extender esta formalización a ciencias empirico-formales como la fisica. Para ello introducen en el formalismo lógico-matemático un conjunto de términos fisicos que precenden poder definir "operacionalmente" mediante terminos "directamente observables". Este sencillo proyecto tropezó con múlti. ples dificultades lógicas ${ }^{6}$. Pero sobre todo fue su despreocupación por la evolución histórica de las ciencias to que le hizo fracasar. El cambio cientifico no pedia explicarse dentro de sus rigidas estructuras lógicas más que por métodos muy artificiosos y parciales, como el reduccionismo de una teoría a otra más elemen- 
tal. Esta preocupación por explicar el cambio cientifi. co. primero a escala históricamente restringida, como un el falsacionismo de Karl Popper ${ }^{\top}$, y luego a escala historicamente amplia, como en las revoluciones cienrificas de Thomas kuhn", es lo que ha dado la pauta a las modernas corrientes epistemológicas. Hoy se ha impuesto la convicción de que, para poder entender las ciencias actuales y apreciar su valor cognoscitivo, es imprescindible considerar su evolución histórica.

\section{LA EPISTEMOLOGIA EVOLUTIVA DE TOULMIN}

En realidad fue Stephen Toulmin quien, desde una amplia visión histórica de las ciencias ${ }^{\circ}$, tuvo el mérito de introducir una episcemologia seriamente inspirada por esa evolución histórica ${ }^{10}$. $\mathrm{Y}$, hace ya diez años publicó bajo el tíulo La comprensión humana" un inte. resante volumen sobre este tema. Su hipotesis fundamental es que "la teoria populacional darwinista de la "variación y selección natural' es un ejemplo de una forma más general de explicación histórica"'12. Con esta explicación histórica aplicada al desarrollo de las ciencias at aca un doble dilema, relacionado con la racionalidad cientifica y con el cambio cientifico.

Con la racionalidad cientifica se relaciona el dilema absolutismo-historicismo. Según el absolutismo, esponáncamente prolesado por grandes racionalistas de Leibnitz a Frege ${ }^{13}$, la racionalidad exige un gran sistema lógico, universal a inmutable. Pero los estudios históricos de las ciencias empirico-formales han hecho perder la confianza en tal racionalidad absolu1a. $Y$ asi, según el historicismo profesado por ejemplo por Collingwood '4, solo cabe hablar de racionalidad dentro de un cierto sistema lógico, evidentemente elaborado con los conceptos de su propio contexto histórico-cultural, y hay que desinteresarse por relacionar sistemas diferentes. Toulmin rechaza tanto el absolutismo de Frege como el historicismo de Collingwood, por presuponer ambos un concepto estático de racionalidad, entendida como atributo de un sistema lógico cerrado. Defiende una racionalidad dinámica, que sea atribulo de la actividad intelectual humana, y cspccialmente de su tarea básica consistente en criticar y modificar tales sistemas conceptuales.

Con el cambio científico se relaciona el dilema uniformismo-revolucionarismo. Segun el uniformismo el cambio cientifico no supone ninguna ruptura, sino un simple progreso acumulativo de unas disciplinas cientificas, que permanecen inmutables con sus mismos métodos y sus mismos objetivos. Según e! revolucionarismo por el contrario esos cambios introducen verdaderas rupturas disciplinares, al establecer nuevos sistemas conceptuales, racionalmente inconmesurables con los anteriores. Veamos la concepción evolutiva que propons Toulmin como via media ante ese dilema uniformismo-revolucionarismo. Prescindiremos de la presentación, a nuestro juicio exagerada, que hace de la "ilusión revolucionaria" de Kuhn, co- mo también del sensacionalismo que da a los descubrimientos de James Cook el 13 de abril de $1769^{15}$.

\section{DIVERSOS NIVELES DE CAMBIO CIENTIFICO}

Dentro de una disciplina cientifica Toulmin distingue tres niveles de contenidos ${ }^{18}$. El primer nivel, ej más externo, es el de las hipótesis, leorias y principios concretos de la disciplina. Un cambio en tales proposicio. nes no significa necesariamente un cambio radical de la disciplina. Pongamos el ejemplo del descubrimien. to de "violación de la paridad", acaecido en 1957 ". Hasta eșa fecha los físicos teóricos solian admitir espontáneamente "Ia conservación de la paridad", o invariancia de las leyes físicas bajo la inversión espacial (es decir, que si un proceso físico es posible según una cierta configuración espacial, to es igualmente según otra configuración, imagen especular de la primera). La conjetura aventurada por T.D. Lee y C.N. Yang en 1956 y experimentaimente comprobada por C.S. Wu y otros en 1957, obligaron a admitir que en ciertos procesos de interacción nuclear débil no se verifica esa invariancia bajo inversión espacial, sino que "la paridad es violada". Muchos físicos teóricos, especialmente Pauli, experimentaron con ello una gran sorpresa. Pero el cambio de ta! principio "se conserva..., o no se conserva la paridad" y aun el cambio de for. mulación que implica para muchas de sus leyes, no supuso un cambio radical para la fisica teorica. Pues su cuadro conceptual quedó prácticamente intacto.

Toulmin considera en las disciplinas cientificas un segundo nivel más profundo, el de la conceptualización. No sólo se refiere a los términos del lenguaje científico, sino también a las técnicas de representación y aun a los procedimientos de aplicasión empirica. Los cambios a este segundo nivel son más profundos. Vienen a corresponder a to que Kuhn llama revoluciones cientificas. En la historia de la física moderna, yo no me atreveria a enumerar más que cuatro de tales cambios radicales que introducen sucesivamente: la mecánica clásica de Galileo y Newton, la teoria de campos de Faraday y Maxwell, la física relativista de Poincaré y Einstein, y la física cuántica de Heisenberg y Bohr. Lo interesante son las diferentes conceptualizaciones que utilizan esos sucesivos cuadros físicos. La mecánica clásica habla de corpúsculos -o agregados de corpúsculosdotados de masa, momento, fuerzas,... La teoria de campos postula en cada punto del espacio. aún del espacio vacio, los cuatro vectores intensidad e inducción eléctricas y magnéticas. La fisica relativista modifica nada menos que los conceptos ordinarios de espacio y tiempo, relacionándolos entre si y con la materia. Y la física cuántica revoluciona el concepto de magnitud fisica $u$ 
"observable", al decir que un mismo sistema cuantico puede tener a la vez - con diversas probabilidades - todos los valores posibles de una magnitud, mientras no se realice una medida de la misma.

Pero según Toutmin en las disciplinas científicas hay todavia un tercer nivel, el más profundo, que permanece vivo bajo tales cambios de conceptualización y da continuidad a las ciencias. Es el nivel de los "ideales explicativos" o las "ambicio. nes intelectuales" que caracterizan cada disciplina e impulsan su correspondiente tarea cientifica. Asi, la "estrategia disciplinar" puede permanecer la misma, aún cuando haya sido preciso variar la "táctica disciplinar", al modificar la con. ceptualización. Creemos interesante esta presentación de la raiz última de las disciplinas cientificas como ideales y ambiciones de carácter profundamente humano y valoral ${ }^{18}$. Pero la inmutabilidad de tales ideales disciplinares, en los cam. bios de conceptualización incluso en los más ra* dicales, es problemática aún para el mismo Toul$\min ^{19}$. Digamos por ejemplo que en la última y más radical de nuestras cuatro revoluciones de la física, el nuevo concepto de "Observable" de tal manera hiere el ideal de objetividad de la descripción fisica, que resulta problemático juzgar si constituye un mero cambio de táctica disciplinar, o supone modificar la estrategia misma de la física.

\section{EL MODELO EXPLICATIVO DARWINIANO}

Centrando la atención en los cambios científicos al segundo nivel de conceptualización, Touimin desarrolla su teoria de la "evolución de los conceptos". Apoyado en su hipótesis evolucionista, aplica aqui el modelo explicativo darwiniano a la historia de las ciencias. Condensa su teoria en cuatro tesis que folmula paralelamente para una "zoologia evolucionista" y para una "gnoseología evolucionista" 2 . En ese paralelismo, a los conceptos zoológicos de "especie" y "población de organismos" corresponden los gnosecológicos de "disciplina" y "población de conceptos".

Según su primera tesis, el modelo darwiniano ha de explicar lanto la continuidad como el cambio de las disciplinas-especies. Estas cobran así el carácter de realidades históricas, disimulado en otras concepciones esencialistas o nominalistas.

Según su segunda tesis darwiniana, continuidad y cambio se explican por el mismo proceso dual de "variación al azar" y "perpetuación selectiva". La rigu. rosa selección critico-natural elimina la mayor parte de las variantes, por resultar desventajosas, y da asi continuidad a la disciplina-especie. Pero al mismo tiempo permite que una pocas innovaciones ventajosas se afiancen y transmitan a las generaciones siguientes.

Según su tercera tesis darwiniana, para un auténtico cambio se exigen tres condiciones: "abundancia de innovaciones" o variantes conceptuales transmisibles, "presión selectiva" que contraste las ventajas y "foro de competencia" adecuado, en el que puedan sobrevi. vir e imponerse las innovaciones ventajosas. Esto viene a constituir una reinterpretación evolucionista del mecanismo de "conjeturas y refutacions" con que Popper sintetiza su falsacionismo.

Según su cuarta tesis darwiniana, bajo los conceptos de "selección" y te "ventajas" se esconde la idea de una verdadera elección razonable. Se eligen las innovaciones que mejor se "adaptan" a las "exigencias" del "nicho ecológico" o medio intelectual local. Esa elección es, en último término, la responsabie de la continuidad y del cambio, y la que hace de las disciplinas-especies entidades históricas en evolución progresiva.

No pretendo que ese paralelismo entre zoologia y gnoseología evolucionistas pueda lievarse al extremo"'. En particular me cuesta ver en las innovaciones con. ceptuales el aspecto irracional de azar. En toda pro. puesta de innovación científica veo más bien un acto profundamente racional, en el que, ante un problema cientifico acuciante, se propone una orientación nueva, muy bien sopesada. Pero creo que ese paralelismo patentiza el carácter profundamente humano e histórico de las disciplinas científicas en su última raíz ra. cional.

\section{RACIONALIDAD DINAMICA DE LAS CIENCIAS}

El uilimo propósito de esta descripción evolutiva de las ciencias consiste en ampliar nuestro concepto de racionalidad. No verla reducida a ta consistencia lógica de un sistema cerrado. Verla más bien en su dinamismo. como atributo de esa empresa profundamente humana, colegial e histórica que es el desarrollo de una disciplina cientifica. La raiz racional de esa actividad cientifica consiste en esas sucesivas elecciones ra. zonables, realizadas colegialmente en los momentos criticos de su cambio histórico. Ellas van modificando, en contacto con experiencias nuevas e imprevistas, los cuadros conceptuales que condicionan todo sistema lógico y toda uiterior racionalidad. Los procedimientos intelectuales que realizan tales modificaciones no pueden ser "estereotipados", programables de antemano. Participan más bien del genio creativo y libre que caracteriza el espiritu humano. Participan también de la inspiración y contraste experimentales. Pero no sólo de la experiencia cientifica inmediata, sino de una experiencia global y colegial que recoja la historia de las ciencias del pasado y aún sea sensible a las expectativas y ambiciones del futuro.
En,

puet

sicic

text!

tan

nos

berit

que

sabe

NOTA

(1) $\mathrm{Cr}_{\mathrm{r}}$

(Con

(2) ANI nom: $e^{\prime \prime}$,

(3) PIER struct. Paris.

(4) HEN? Paris Caipe

(5) VIC

(6) DIEC cia: mo 0

(7) KARI I935. nos (E

(8) THOA Univel de las (Breve

(9) STEPI son, $I$ Londo The Di. The Ar. ENSENANZA DE LAS CIENCIAS 
En nuestro mundo didóctico tal vision de las ciencias puede proporcionar un buen antidolo contra la exposición dogmática, tan frecuente en nuestros libros de texto, y contra la caricatura cientista de racionalidad, tan difundida e idolatrada sobre todo por los profa. nos de las ciencias. Nuestros alumnos de ciencias de. berian oir de los zanteos, los fracasos y los exitos con que va progresando la empresa cientifica. Y deberian saber que los fracasos - necesarios para posibilitar los éxitos - no siempre se han debido a prejuicios an. ticientificos, sino normalmente a complejidad de la naturaleza y a limitación de nuestro entendimiento. Esto les podria hacer ver que también las ciencias actuales exigen una tarea, profundamente humana, de critica y progreso. Y aún podria suscitar entre ellos verdaderas vocaciones cientificas que, una vez maduradas, se incorporen a esa tarea profundamente hu. mana.

\section{NOTAS Y BIBLIOGRAFIAS}

(1) Cfr. FRANCIS BACON, "Novum Organtum", Fontanelia (Colección Clásicos de Filosofia S47), Batcelona 1979.

(2) ANDRE-MARIE AMPERE, "Théorie mathématique des phénomenes electro-dynomiques uniquement deduite de lexperien. ce". Paris 1827. Reedición: Qlanchard, Paris 1958.

(3) PIERRE DUHEM, "La Theorie Physique: Son objet et sa structure", Chevalier et Rividre, Paris 1906 . Reedicion: Vrin, Paris 1981 .

(4) HENRI POINCARE, La Science et I'Hypothèse. Flammarion, Paris 1902. Traducción: Lo Ciencia y la Hipotesis. EspasaCalpe (Colección Austral), Madrid 1963

(S) VICTOR KRAFT, EI Circulo de Viena. Taurus, Madrid 1966.

(6) DIEGO RIBES "Panorámica actual de la filosofia de la cien. cia: Estructura incerna de teorias y cambio cientifico". Teorema 6 (1306), 359-425.

(7) KARL R. POPPER, Lagik der Forschung, Springer. Wien 1935. Traducción: Le logica de lo invessigación cientffica. Tecnos (Estructura y función, 8). Madrid 1962.

(8) THOMAS S. KUHN. The Siructure of Scientific Revolutions, University of Chicago Press, 1962 . Traduccion: Le estrucrura de las revoluciones cientificas, Fondo de Cultura Económica (Breviarios, 213), Mixico 1971 .

(9) STEPHEN TOULMIN, rhe Fabric of the Heavens. Hutchin. son. London 1961; The Archilecture of Motier. Hutchinson. London 1963; STEPHEN TOULMIN y JUNE GOODFIELD. The Discovery of Time, Hutchinson, London 1965 (Coleccion: The Ancestry of Science).
(10) STEPHEN TOULMIN, The Philosophy of Science, Harper and Row, London 1953: La filosofio de la ciencia, Fabris, Bucnos Aires 1964; Foresight ond Understanding, Hutchinson. London 1961 .

(1) STEPHEN TOULMIN, Human Undersianding, vol. I: The Collective Use and Evolution of Concepts, Princeton University Press 1972. Traducción: La comprension humana, l: El uso colectivo y la evolución de los conceptos, Alianza Editorial (AU 191), Madrid 1977

(12) lbidem, pág. 145 de ta edición castellana

(13) GOTTLOB FREGE. The Foundations of Arithmetic, Breslau 1844

(14) R.G. COLLINGWOOD. An Essay on Meraphysics, Oxford 1940.

(15) Cf. referencia 11 , págs. 107-132 y págs. $55-63$ de la edición castellana.

(16) ibidem. págs. 155-198.

(17) Cf. MANUEL GARCIA DONCEL. porticules, compas y simesrias: Historia de la Flsica de Altas Energfas de los aflos $30_{a}$ los 60. Servicio de Publicaciones de la Universidad Attonoma de Barcelona (UAB-HEF.D2). Bellaterra 1982, págs. 188-192.

(18) Cf. THOMAS S. KUHN, The Essential Tension. University of Chicago Press, 1977, capitulo 13: "Objectivity Value Judgment, and Theory Choice", pags. 320-339.

(19) Cf. referencia 1!, págs. 24]-247 de la edición casteliana.

(20) lbiden págs. 145-ls!

(21) Cr. 1. IONATHAN COHEN, "ls the Progress of Science Evolutionary?". British Journal for the philosophy of Science. vol. 24 (1973), pags. 4i-6i. 